\title{
Musik als Wissenschaft im Raum der Personalunion
}

\author{
Andreas WaczKat
}

Am 23. Juni 1733 vermerkt die Londoner Tageszeitung „The Bee“:

Great Preparations are making for Mr. Handel's Journey to Oxford, in order to take his Degree of Musick; a Favour that University intends to compliment him with, at the ensuing Publick Act. The Theatre there is fitting up for the Performance of his Musical Entertainments, the first [of] which begins on Friday Fortnight the $6^{\text {th }}$ of July. We hear that the Oratorios of Esther and Deborah, and also a newer one never performed before, called Athaliah, are to be represented two Nights each; and the Serenata of Acis and Galathea as often. ${ }^{1}$

Keine Frage: spricht man über Musik im Raum der Personalunion, zieht die Person Georg Friedrich Händels die mit Abstand größte Aufmerksamkeit auf sich. Dass der vormalige hannoversche Hofkapellmeister indes auch - zumindest in gewisser Weise - für die Geschichte und das Verständnis der Musik als Wissenschaft im Raum der Personalunion zentral ist, belegt dieses Ereignis aus dem Jahr 1733. Die Universität Oxford, begründet im 12. Jahrhundert und damit eine der ältesten Universitäten Europas, beabsichtigte, Händel den akademischen Grad eines Doctor Musicae - D.Mus. - zu verleihen; ein Grad, der wie auch der des Baccalaureus Musicae - B.Mus. - wohl seit dem frühen 16. Jahrhundert vergeben worden war. Händel sollte anlässlich dieser Ehrung, so berichtet es „The Bee“, und so bestätigen es die Zeitungen aus Oxford, seine Oratorien Esther, Deborah und die eigens zu diesem Anlass komponierte Athalia aufführen, ebenso die unterhaltende kleine Seranata Acis und Galathea. Händel hatte dazu, so berichtet "The Bee“ weiter, auch die entsprechenden Gesangssolisten aus London in die wohl eine Tagesreise entfernte Universitätsstadt mitgenommen: die

1 Zitiert nach: Walter Eisen (Hg.), Händel-Handbuch, Bd. 4, Dokumente zu Leben und Schaffen, Leipzig 1985, S. $215 f$. 
Sopranistinnen Anna Strada und Mrs. Wright, die beiden Tenöre Thomas Salway und Philip Rochetti sowie den Bass Gustavus Waltz.

Die Aufführungen fanden allgemeines Lob, und sie überstiegen die Anforderungen, die die Universität Oxford an die Verleihung der akademischen Grade des Baccalaureus oder des Doctor Musicae geknüpft hatte, bei weitem. Der Baccalaureus Musicae konnte solchen Personen verliehen werden, die sich sieben Jahre nachweislich dem Studium und der Praxis der Musik verschrieben hatten und ein fünfstimmiges Canticum zum Examen vorlegten, das auch in der School of Music der Universität Oxford mit Vokalstimmen und Instrumenten aufzuführen war. ${ }^{2}$ Mit einem solchen Canticum ist ein einsätziges geistliches Werk gemeint, etwa ein einfaches Anthem, wie es im Rahmen einer gottesdienstlichen Feier in der anglikanischen Kirche vorkommt. Für die sehr viel seltenere Verleihung des Doctor Musicae bedurfte es fünf weiterer Jahre der praktischen oder theoretischen Auseinandersetzung mit der Musik; das vorzulegende Canticum wurde nun sechs- oder achtstimmig erwartet. ${ }^{3}$ Mit den insgesamt zwölf Jahren des Studiums der Musik ist freilich keine universitäre Ausbildung gemeint: Die Musik war kein Studienfach an den Universitäten. Vielmehr hatten die Kandidaten für diesen Titel ihre jeweilige Beschäftigung mit der Musik durch geeignete Nachweise zu belegen. William Croft beispielsweise, dem der Titel des Doctor Musicae im Jahr 1713 verliehen worden war, konnte das leicht tun: Nachdem er einige Jahre als Chorknabe in der Chapel Royal gesungen hatte, wurde er im Jahr 1700 Organist an der St. Anne's Church in London, später Musiklehrer an der Chapel Royal, 1708 schließlich Organist an der Westminster Abbey. ${ }^{4}$ In dieser Stellung komponierte er 1714 - also schon als Doctor Musicae - sowohl die Musik für die Begräbnisfeierlichkeiten von Queen Anne als auch die Musik für die Krönungszeremonie für den hannoverschen Kurfürsten Georg Ludwig zum King George I. Croft steht auf diese Weise an führender Stelle, wenn es um Musik im Raum der Personalunion geht. Er hatte also schon 13 Jahre einer professionellen Karriere hinter sich, und auch das Canticum, mit dem er sich der Prüfung unterzog, ist bekannt: Das Anthem This is the day, komponiert anlässlich des Friedens von Utrecht im Jahr 1713, ${ }^{5}$ für den auch schon Händel das Utrechter Te Deum geschaffen hatte.

Im Vergleich zu Händels abendfüllendem Oratorium Athalia ist Crofts Promotionsanthem ein klein dimensioniertes Werk, und bei aller souveränen Beherrschung des Kompositionshandwerks fehlt es Crofts Kompositionen doch an jenem zündenden Feuer, mit dem die Händelschen Oratorien so schnell für sich einnehmen, wofür

2 Susan Wollenberg, Music in 18th-Century Oxford, in: Proceedings of the Royal Music Association 108 (1981/82), S. 69-99, hier S. 70.

3 Wollenberg, 1981/82 (wie Anm. 2), S. 71.

4 Watkins Shaw, William Croft, in: Ludwig Finscher (Hg.), Die Musik in Geschichte und Gegenwart (im Folgenden MGG), Personenteil 5, Kassel 22001, Sp. 120-122, hier Sp. 120.

5 Shaw, 2001 (wie Anm. 4). 
auch Athalia ausgezeichnete Beispiele parat hält. Händel hatte also alle Bedingungen für die Verleihung des Doctor Musicae erfüllt - und lehnte den Titel dennoch ab. Was war passiert? Über die Gründe lassen sich nur Mutmaßungen anstellen, denn in den zahlreichen Dokumenten, die im Zusammenhang mit dieser Titelverleihung einschlägig sind, ${ }^{6}$ finden sich dazu keine Hinweise. Immer wieder einmal wird daher gemutmaßt, dass Händels Bescheidenheit ihn zur Ablehnung des Titels geführt haben könnte, ${ }^{7}$ doch beruht das wohl auf einer biographischen Projektion. Der einzige Komponist, dem schon zu Lebzeiten ein Denkmal gesetzt worden ist, nämlich die 1737/38 von Louis François Roubiliac für die Vauxhall Gardens geschaffene Statue, hat es ja auch akzeptiert, dabei als Apollo dargestellt zu werden.

Wahrscheinlicher sind damit zwei andere Gründe: Zum Einen wird angeführt, dass Händel die Prüfungsgebühr in Höhe von $£ 100$ zu hoch gewesen sein könne, ${ }^{8}$ was zwar denkbar, angesichts des von der Norwich Gazette am 21. Juli 1733 berechneten Reingewinns von $£ 2.000^{9}$ aus den in Oxford gegebenen Konzerten aber nicht sehr überzeugend ist. Zum Anderen war der Doctor Musicae 1715 auch an den Organisten Maurice Greene verliehen worden, der seit 1730 Professor der Musik an der Universität Oxford war. Greene allerdings trat um 1715 auch für Händel die Bälge, wenn dieser an der Orgel der St. Paul's Cathedral spielte. Auf Augenhöhe mit Händel stand der Herr Professor Greene also sicherlich nicht, wenngleich er gewiss auch ein solider Komponist war.

Für das Nachdenken über Musik als Wissenschaft im Raum der Personalunion steckt diese Episode allerdings schon ganz wesentliche Punkte des Rahmens ab. Nicht die Residenzstädte sind es, die die Aufmerksamkeit auf sich ziehen, sondern die Universitäten Oxford und Göttingen. Und es geht um die Entdeckung der Beschäftigung mit Musik als theoretischer Disziplin eigenen Rechts, wie es sich beispielsweise auch an der Verleihung des Oxforder Doctor Musicae an Samuel Arnold im Jahr 1773 ablesen lässt. Zwar hatte auch Arnold, der später noch Hofkomponist, Direktor der Academy of Ancient Music sowie Organist an der Westminster Abbey werden sollte, ${ }^{10}$ eine professionelle Karriere als Musiker noch vor sich, doch wesentlich in Erscheinung getreten ist er als Herausgeber der ersten Gesamtausgabe der Werke Händels,

6 Eisen, 1985 (wie Anm. 1), S. 218-221.

7 Eisen, 1985 (wie Anm. 1), S. 220, jedoch ohne Nennung spezifischer Biographen. Der mutmaßlicherweise früheste Beleg für Händels angebliche Bescheidenheit findet sich bei Friedrich Chrysander, G. F. Händel, Leipzig 1858, S. 87. Allerdings spielen die Umstände der Oxforder Promotion bei Chrysander keine Rolle.

8 Eisen, 1985 (wie Anm. 1), außerdem Hans-Joachim Marx, Georg Friedrich Händel, in: Ludwig Finscher (Hg.), MGG, Personenteil 8, Kassel 22002, Sp. 509-638, hier Sp. 529.

9 Eisen, 1985 (wie Anm. 1), S. 221.

10 Robert Hoskins, Samuel Arnold, in: Ludwig Finscher (Hg.), MGG, Personenteil 1, Kassel 21999, Sp. 1002-1008, hier Sp. 1003. 
was von Robert Hoskins als „hauptsächlich wissenschaftliche Arbeit ${ }^{\text {"11 }}$ charakterisiert wird.

Diese Entwicklung hat eine ebenso lange Vor- wie Nachgeschichte. In Oxford bietet es sich an, zunächst in das Jahr 1626 zurückzuschauen, denn in diesem Jahr wurde von William Heather die Professur für Musik gestiftet, deren als nicht miteinander vereinbar angesehene Aufgaben in der Theorie und Praxis der Musik von zwei Personen wahrgenommen wurden: einem Chorleiter, der die allwöchentlichen Proben des Universitätschors zu leiten hatte, und einem Lektor, der in Fortsetzung der mittelalterlichen und frühneuzeitlichen Tradition die theoretische Beschäftigung mit der Musik im Rahmen des Quadriviums anzuleiten, mithin Musiktheorie als Zahlwissenschaft zu betreiben hatte. ${ }^{12}$ Dabei zeigt schon das Gehalt der beiden Personen die klare Hierarchie auf: Der Lektor bezog $£ 3$ jährlich, der Chorleiter mit $£ 13$ aber mehr als das Vierfache. Noch im 17. Jahrhundert blieb die Position des Lektors dauerhaft vakant, während der Chorleiter seit dem frühen 18. Jahrhundert schon den Titel Professor trug. Hier allerdings liegt auch der Grund dafür, dass der Baccalaureus und der Doctor Musicae ohne vorangegangenes Universitätsstudium verliehen werden konnten, wenn nicht mussten: Der Professor of Music hielt gar keine Vorlesungen.

Und so waren die meisten Träger eines der musikalischen akademischen Grade in Oxford Organisten, Chorleiter und Komponisten. Zum Doctor Musicae ernannt wurde beispielsweise neben den bereits erwähnten William Croft und Maurice Greene im Jahr 1713 Johann Christoph Pepusch, ein Pfarrerssohn aus Berlin, der sich schon seit 1704 in London als Komponist etabliert hatte; er ist auch einer der Mitbegründer der Academy of Ancient Music in London. 1729 wurde John Stanley zum Doctor Musicae promoviert, ein blinder Organist und Geiger, Schüler von Maurice Greene und später Direktor des Londoner Foundling Hospital. 1785 erwarb Friedrich Hartmann Graf diesen Titel. Graf war eigentlich Kantor und Musikdirektor in Augsburg, in den 1780er-Jahren indes Dirigent der von dem Mannheimer Komponisten Johann Wilhelm Cramer ins Leben gerufenen Professional Concerts in London, wo sich Graf offenbar einer sehr großen Wertschätzung erfreuen konnte. 1794 erhielt der zu diesem Zeitpunkt gerade 18-jährige William Crotch den Titel eines Doctor Musicae. Das Kriterium einer bereits zwölf Jahre umfassenden professionellen Karriere scheint hier zunächst schwer erfüllbar, doch soll Crotch schon als Zweijähriger auf einer Orgel die britische Nationalhymne gespielt haben, und schon seit 1779, als Vierjähriger, konzertierte er täglich öffentlich. ${ }^{13}$ Der britische Musikhistoriker

11 Hoskins, 1999 (wie Anm. 10).

12 Wollenberg, 1981/82 (wie Anm. 2), S. 70.

13 Jonathan Rennert, William Crotch, in: Ludwig Finscher (Hg.), MGG, Personenteil 5, Sp. 135-140, hier Sp. 136. 
Charles Burney referierte am 18. Februar 1779 vor der Royal Society über diese außergewöhnliche Frühbegabung. ${ }^{14}$

Charles Burney, Doctor Musicae der Universität Oxford seit 1769, war 1726 in Shrewsbury in den West Midlands als Sohn eines Porträtmalers geboren worden. ${ }^{15}$ Nach dem Besuch der Public School in Chester wurde er 1744 Schüler des Komponisten Thomas Arne in London; hier spielte Burney auch Violine und Viola in jenem Orchester, mit dem Händel seine Oratorienaufführungen bestritt. 1749 wurde er Organist an der St. Dionis Backchurch. Nachdem er sich 1751 aus gesundheitlichen Gründen nach Norfolk zurückgezogen hatte, kehrte er mit seiner Familie, zu der auch seine erste Tochter Esther als begabte Cembalistin gehörte, 1760 nach London zurück, wo er zunächst vornehmlich als Konzertveranstalter, aber auch als Komponist wirkte.

1774 verfolgte Burney den Plan der Gründung einer Music School, 1783 wurde er zum Organisten am College in Chelsea ernannt, wo er 1814, ausgestattet mit einer Staatspension starb. Nachdem er den Oxforder Doctor Musicae erhalten hatte, bereiste er ab 1770 Frankreich, Italien, Deutschland und die Niederlande, um Material für ein großes Vorhaben zu sammeln, das ihn wohl schon seit den 1750er-Jahren beschäftigt hatte: Burney wollte eine Geschichte der Musik schreiben. Aus seinen Reisen resultierte jedoch weit mehr: Im Jahr 1771 erschien sein Buch „The Present State of Music in France and Italy ", ${ }^{16}$ im Jahr 1773 folgte Burneys zweibändige Abhandlung „The Present State of Music in Germany, the Netherlands, and United Provinces "17; alle drei Teile wurden von Christoph Daniel Ebeling und Johann Joachim Christoph Bode als „Carl Burney's der Musik Doctors Tagebuch seiner Musikalischen Reisen " übersetzt und noch im selben Jahr gedruckt. ${ }^{18}$ Auch die projektierte Geschichte der Musik ließ nicht sehr lange auf sich warten, 1776 erschien der erste Teil einer insgesamt vierteiligen „General History of Music from the Earliest Ages to the Present Period“, deren folgende drei Teile von 1782 bis 1789 herauskamen. ${ }^{19}$ Aus

14 Charles Burney, Account of an Infant Musician, London 1779.

15 Jürgen Mainka, Charles Burney, in: Ludwig Finscher (Hg.), Personenteil 3, Sp. 1319-1326, hier Sp. 1319 ff.

16 Charles Burney, The Present State of Music in France and Italy or, the Journal of a Tour through those Countries, undertaken to collect Materials for a General History of Music, London 1771.

17 Charles Burney, The Present State of the Music in Germany, the Netherlands and the United Provinces or, the Journal of a Tour through those Countries, undertaken to collect Materials for a General History of Music, 2 Bde London 1773.

18 Carl Burney's der Musik Doctors Tagebuch einer musikalischen Reise durch Frankreich, Italien, Flandern, die Niederlande und am Rhein bis Wien, durch Böhmen, Sachsen, Brandenburg, Hamburg und Holland 1772/1773, welche er unternommen hat um zu einer allgemeinen Geschichte der Musik Materialien zu sammlen, Hamburg 1772-1773.

19 Charles Burney, A General History of Music From the Earliest Ages to the Present Period, London 17761789. 
dem ersten Band erlebte der vorangestellte Abschnitt über die Musik der Antike auch eine deutsche Übersetzung in Gestalt von „Dr. Karl Burney’s Abhandlung über die Musik der Alten“, die Johann Joachim Eschenburg, Professor für Englische Sprache am Braunschweiger Collegium Carolinum für den Leipziger Verlag Schwickert angefertigt hatte. ${ }^{20}$ Eschenburg übersetzte auch noch eine zweite Schrift Burneys, nämlich dessen „Account of the musical performances in Westminster Abbey and Pentheon in commemoration of Handel", ${ }^{21}$ in der Burney die Feierlichkeiten anlässlich von Händels 25. Todestag ausführlich beschreibt und kommentiert: vornehmlich die Aufführung von Händels Oratorium Messiah in Anwesenheit der königlichen Familie mit nicht weniger als 513 Mitwirkenden.

Mit seiner "General History of Music“ freilich hat Burney eine Arbeit vorgelegt, die ungeachtet zahlreicher Fehler im Detail der theoretischen Beschäftigung mit Musik eine neue Perspektive erschlossen hat, nämlich die historische. Burney ist dabei sicherlich in vielerlei Hinsicht beeinflusst gewesen von dem Gedanken, der auch vielen anderen Schriften des 18. Jahrhunderts zugrunde liegt, die unter Titeln wie „General History of the World“ - z. B. von William Guthrie 1764-1767 publiziert $^{22}$ - oder „History of Mankind“ - z. B. von James Dunbar $1780^{23}$ - erschienen sind. Während diese genannten Schriften aber allesamt mehr oder weniger zahlreiche Vorläufer haben, war Burney in gewisser Weise Pionier. Einzig der italienische Franziskaner-Mönch Giovanni Battista Martini hatte mit seiner „Storia della Musica“, die zwischen 1757 und 1781 in Bologna verlegt wurde,${ }^{24}$ sowie der Londoner Jurist John Hawkins mit seiner 1776 in gleich fünf Bänden erschienenen „A general history of the science and practice of music" 25 traten hier, allerdings annähernd gleichzeitig, hervor. Gemeinsam ist diesen Schriften nicht allein die historische Perspektive und die grundsätzliche Erkenntnis, dass Musik überhaupt ein Gegenstand ist, der sich historisch fassen und untersuchen lässt, sondern auch, dass die gewählten Methoden bis in die Gegenwart hinein immer noch einen Teil des Methodenbestands musikwis-

20 Dr. Karl Burney's Abhandlung über die Musik der Alten. Aus dem Englischen übersetzt, und mit einigen Anmerkungen begleitet von Johann Joachim Eschenburg, Leipzig 1781.

21 Charles Burney, An Account Of The Musical Performances In Westminster-Abbey, And The Pantheon [...] 1784, in: Commemoration Of Handel, London 1785. Übersetzt als: Dr. Karl Burney's Nachricht von Georg Friedrich Händel's Lebensumständen und der ihm zu London im Mai und Juni 1784 angestellten Gedächtnißfeyer. Mit Kupfern aus dem Englischen übersetzt von Johann Joachim Eschenburg, Berlin / Stettin 1785.

22 William Guthrie, A General History of the World from the Creation to the Present Time, 12 Bde, London 1764-1767. Guthries General History of the World ist die gewissermaßen konsequente Ausweitung seiner dreibändigen General History of England, London 1744-1751, wie auch seiner zehnbändigen General History of Scotland, London 1767-1768.

23 James Dunbar, Essays on the History of Mankind in Rude and Cultivated Ages, London 1780.

24 Giovanni Battista Martini, Storia della Musica, 3 Bde, Bologna 1757-1781.

25 John Hawkins, A General History of the Science and Practice of Music, 5 Bde, London 1776. 
senschaftlicher Forschung ausmachen, insofern Textquellen herangezogen werden, die nach philologischen Kriterien im Hinblick auf die enthaltenen Informationen über die Geschichte der Musik befragt werden. Dass dabei von Martini, Burney und Hawkins im Streben nach Universalität der Darstellung auch Kapitel geschrieben worden sind, die nahezu frei von Tatsachen sind, hat Alexander Rehding unlängst am Beispiel von Burneys Darstellung der Musik im alten Ägypten gezeigt; ${ }^{26}$ dies mindert jedoch nicht den Wert der grundsätzlichen Einsicht, dass es auch im alten Ägypten eine Musik gegeben hat, über die sich berichten lässt, um damit einen Beitrag zum allgemeinen Weltwissen zu leisten.

John Hawkins trat mit seiner "General history of the science and practice of music" gleichzeitig mit Burney in Erscheinung, wobei Hawkins vier Monate später als Burney allerdings schon seine komplette Abhandlung veröffentlichte, während von Burneys „General History“ zunächst nur der erste Teil in die Buchhandlungen gekommen war, der die Musik im alten Ägypten, im alten Israel, im antiken Griechenland sowie im antiken Rom behandelt. Hawkins war allerdings nicht zuletzt persönlich in einer günstigeren Position. Geboren 1719 in London, hatte er Jura studiert und im Anschluss bis 1753 als Rechtsanwalt und Richter in verschiedenen Ämtern gearbeitet. ${ }^{27}$ In diesem Jahr aber heiratete er Sidney Storer, eine sehr wohlhabende Frau, deren Vermögen es Hawkins erlaubte, sich aus allen Ämtern zurückzuziehen und als Privatier wohl über 16 Jahre hinweg an seiner „General History of the Science and Practice of Music" zu schreiben. 1772 in den Adelsstand erhoben, starb er 1789 in London wenige Wochen bevor die letzten Bände von Burneys „General History“ aus der Druckerei kamen. Allerdings: den Doctor Musicae der Universität Oxford hat er nie erhalten. Hawkins war musikalischer Laie, ein Amateur im besten Sinn des Wortes, der zwar Mitglied in einer singing society war, jedoch nicht in der Lage gewesen wäre, ein Canticum zu komponieren.

Doch das ist sicher nicht der einzige Grund, warum schon damals Burneys „General History" eindeutig vorgezogen und auch sehr viel besser verkauft wurde. Burney hatte Europa zumindest in Teilen bereist und Informationen aus erster Hand von Gewährspersonen gesammelt, wo Hawkins all sein Wissen am Schreibtisch aus anderen Büchern bezog. Schon Burney selber verspottete Hawkins Konkurrenzprodukt in einer bösen, allerdings damals ungedruckt gebliebenen Satire:

Around Apollo's radiant throne, a crowd

Has late assembled, clamorous and loud;

Complaining of a certain scribe malign

26 Alexander Rehding, Die ägyptische Spieldose, in: Sandra Danielczyk u. a. (Hg.), Konstruktivität von Musikgeschichtsschreibung, Hildesheim 2012, S. 11-33, hier S. 13 f.

27 Bernhard Schrammek, Sir John Hawkins, in: Ludwig Finscher (Hg.), MGG, Personenteil 8, Kassel 22002, Sp. 895-898, hier Sp. 895f. 
Unlicens'd by the God, or Muse divine;

Unauthoriz'd by Judgment, Talents, Taste

Unprincipl'd in present Lore or past;

Without an Ear to hear, or soul to feel,

Without a mask his malice to conceal. ${ }^{28}$

Die Rivalität von Burney gegen Hawkins - in anderer Richtung ist davon nichts zu erkennen - spiegelte sich aber ohnehin in vielen Kontexten. So ist ein damals populärer Kanon - ein catch, eine besondere Form der musikalischen Satire - bekannt, in der ganz typisch mit Wortspielen die Komik transportiert wird:

Sir John Hawkins

Burn 'is History

How d'ye like him?

Burn 'is History

Burney's History pleases me. ${ }^{29}$

Gerecht wird man Hawkins und seiner „General History“ mit diesen Versen sicher nicht, und so hat Robert Stevenson schon 1950 in einem Aufsatz formuliert:

"Hawkins deserves a better reputation than they bestowed upon him; and students of his career nowadays rate him among the three great pioneers of modern musicology. A century before musicological endeavor flowered, Sir John by some proleptic insight grasped the basic principles of a discipline that has only now come into its own." 30

Dass die historischen Perspektiven the basic principles der Musikwissenschaft seien, war zwar selbst in der britischen Sichtweise um 1950 kein eindeutiger Konsens und ist es heutzutage noch um ein Vielfaches weniger, doch worauf Stevenson in seiner apologetischen Würdigung nicht zu Unrecht hinweist, ist die Bedeutung der grundsätzlichen Erkenntnis, dass Musik ein historischer Gegenstand ist.

Daran allerdings haben Burney und Hawkins gleichermaßen Anteil. Wer aber ist für Stevenson der dritte Pionier der modernen Musikwissenschaft? Es ist nicht Martini mit seiner „Storia della Musica“, sondern es ist der Göttinger akademische Musikdirektor Johann Nikolaus Forkel. Forkel, geboren 1749 in Meeder bei Coburg, war für kurze Zeit Schüler des Lüneburger Johanneums gewesen, bevor er schon als 17-Jähriger zum Präfekt des Schweriner Domchors berufen wurde. 1769 stattete

28 Zitiert nach Robert Stevenson, “The Rivals" - Hawkins, Burney, and Boswell, in: The Musical Quarterly 36 (1950), S. 67-82, hier S. 72.

29 Stevenson, 1950 (wie Anm. 28), S. 74 f.

30 Stevenson, 1950 (wie Anm. 28), S. 82. 
ihn sein Dienstherr, der mecklenburgische Herzog Friedrich, genannt der Fromme, mit einem Stipendium aus, mit dem Forkel in Göttingen Jura studieren sollte. Forkel betrieb diese juristischen Studien wohl nur mit begrenztem Engagement. Wenngleich der Mecklenburgische Herzog ihn bereits für den Hofdienst in Schwerin bzw. Ludwigslust bestimmt hatte, schien sich Forkel an der Göttinger Universität schnell etablieren zu wollen: Schon von 1770 an verwaltete er für zunächst drei Jahre das Organistenamt an der Universitätskirche. ${ }^{31}$

Der junge Student Forkel interessierte sich freilich nicht nur für die Praxis der Musik. In seiner späteren „Allgemeinen Litteratur der Musik“, einer 1792 erschienenen Bibliographie des musikalischen Schrifttums, bekundete Forkel, bereits in diesen Jahren um 1770 eine Kenntnis der wichtigsten musikalischen Schriften über seinen kleinen Büchervorrat,

worunter sich außer den meisten deutschen und einigen ausländischen Lehrbüchern, auch der erste Band von der Storia della Musica des P. Martini, Walthers mus. Lexicon, Adlungs Anleitung zur mus. Gelahrtheit, nebst den bekannten Zeitschriften von Mattheson, Mitzler, Marpurg und Hiller befanden, ${ }^{32}$

erlangt zu haben. Mit diesem nach eigenem Bekunden defizitären Wissen ausgestattet, wagte sich Forkel schon drei Jahre nach seiner Immatrikulation an ein ambitioniertes Projekt: Im Winterhalbjahr 1772 kündigte er privatim Vorlesungen über die Theorie der Musik an. Diese Vorlesungen verstanden sich als wissenschaftliche Veranstaltungen, für die das akademische Umfeld der Universität Göttingen günstige Voraussetzungen bot; tatsächlich hatte Forkel hier Gegebenheiten und Gelegenheiten passgenau zueinander gebracht und etwas getan, was noch anderthalb Jahrhunderte später so vertraut schien, dass Heinrich Edelhoff in seiner 1935 in Göttingen gedruckten, jedoch in Freiburg entstandenen Dissertation ${ }^{33}$ Forkel zum „Begründer der Musikwissenschaft als einer modernen Hochschuldisziplin“ erklärte, und auch Axel Fischer würdigte Forkels Vorlesungen noch als „Keimzelle der musikwissenschaftlichen Universitätslehre “. ${ }^{34}$

Diese Sichtweise hat Manches für sich. Forkel war offenbar ein weitsichtiger und vielseitig interessierter Denker, der sich, ohne sich dabei auf allzu viele Vorbilder berufen zu können, für viele Arbeitsbereiche engagierte, die heute der Musikwissenschaft zugerechnet werden, und der zudem in seinen einschlägigen Schriften auch

31 Axel Fischer, Johann Nikoslaus Forkel, in: Ludwig Finscher (Hg.), MGG, Personenteil 6, Sp. 1458-1468, hier Sp. 1458ff.

32 Johann Nikolaus Forkel, Allgemeine Literatur der Musik, Leipzig 1792, S. V.

33 Heinrich Edelhoff, Johann Nikolaus Forkel. Ein Beitrag zur Geschichte der Musikwissenschaft, Göttingen 1935, S. 102.

34 Fischer, 2001 (wie Anm. 31), Sp. 1462. 
theoretisch über die Fragestellungen und Methoden reflektierte. Zu seinen Tätigkeitsfeldern zählten die Musikhistoriographie ebenso wie die Komponistenbiographik, die Lexikographie und die Editionsphilologie. Forkel übersetzte musikbezogene Schriften aus dem Italienischen, dem Französischen und dem Lateinischen, er rezensierte umfangreich die aktuellste Forschungsliteratur für die „Göttingischen Anzeigen von gelehrten Sachen“.

Im Februar 1779 wurde Forkel zum Akademischen Musikdirektor der Universität ernannt. Zu seinen Aufgaben gehörten die Leitung des Collegium Musicum und die Organisation der Akademischen Winter-Concerte. Anlässlich des 50jährigen Stiftungsjubiläums der Göttinger Universität wurde ihm 1787 die Ehrendoktorwürde verliehen - den Titel eines Doctor Musicae gab es hier nicht. Nachdem sich Forkel 1789 erfolglos um die Nachfolge Carl Philipp Emanuel Bachs als Musikdirektor der Hamburger Hauptkirchen beworben hatte, blieb er der Universität als Musikdirektor bis zu seinem Tod 1818 erhalten.

In den Anfängen seiner akademischen Lehrtätigkeit ging Forkel noch einen anderen Weg der Auseinandersetzung mit Musik als Burney, Hawkins oder der von Forkel auch erwähnte Martini. Die gedruckte Einladungsschrift zu Forkels Vorlesung über die Theorie der Musik gibt darüber Auskunft. Forkel behandelte

die physikalische Klanglehre,

die mathematische Klanglehre,

musikalische Grammatik mit ihren Teildisziplinen,

der musikalischen Zeichenlehre,

den musikalischen Tonarten,

der Lehre von der Harmonie,

und der Rhythmodie,

die musikalische Rhetorik mit ihrer Teildisziplin,

der musikalischen Periodologie, und schließlich

die musikalische Kritik. ${ }^{35}$

Dass dieser Theorie der Musik auch eine Geschichte der Musik an die Seite wuchs, war gewissermaßen konsequent, denn Forkel reagierte damit auf das erwachende Interesse an allgemeiner Musikgeschichte auch in Göttingen, wo August Ludwig Schlözer mit seiner „Vorstellung einer Universal-Historie“ 1772 einen ähnlichen Grund legte, ${ }^{36}$ wie ihn auch Burney und Hawkins in Großbritannien gefunden hatten. Die Göttinger Universität als Universität der Personalunion ermöglichte hier eine sehr

35 Johann Nikolaus Forkel, Ueber die Theorie der Musik, insofern sie Liebhabern und Kennern nothwendig und nützlich ist. Eine Einladungsschrift zu musikalischen Vorlesungen, Göttingen 1777, S. 12-28.

36 Ludwig August Schlözer, Vorstellung seiner Universal-Historie, 2 Bde, Göttingen 1772-1773; zum Einfluss Schlözers auf Forkel vgl. auch Frank Hentschel, Modularisierte Musikgeschichte, in: Sandra Danielczyk u. a. (Hg.), Konstruktivität von Musikgeschichtsschreibung, Hildesheim 2012, S. 241-260, hier S. 243. 
enge Anlehnung, denn die einschlägigen englischen Schriften waren schnell auch in der Göttinger Universitätsbibliothek verfügbar: Die „General Histories“ von Hawkins und Burney sogar schon unmittelbar nach ihrem Erscheinen.

Dem Vorwort in der späteren „Allgemeinen Literatur der Musik“ zufolge begann sich Forkel zwischen 1776 und 1777 besonders für die Geschichte der Musik zu interessieren. ${ }^{37}$ Das ist also genau die Zeit vor seiner zweiten und dann dauernden Vorlesungstätigkeit an der Göttinger Universität und dem Erscheinen der „General Histories" von Hawkins und Burney, die Forkel ausweislich des Registers der Universitätsbibliothek vielfach ausgeliehen hat. ${ }^{38}$

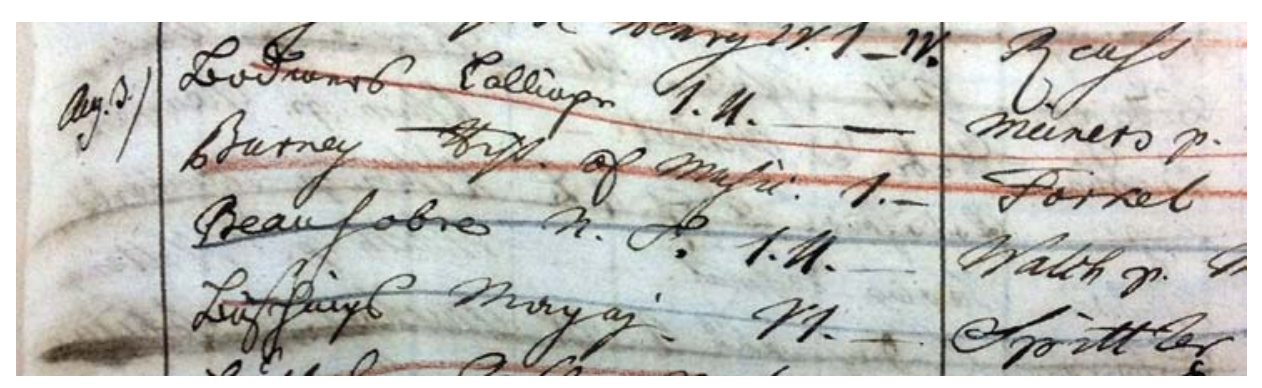

Abb. 1: Archiv der Niedersächsischen Staats- und Universitätsbibliothek, Ausleihregister Ostern 1783-Ostern 1784, Eintrag vom 3. August 1783: Forkel leiht zum wiederholten Mal den ersten Band von Burneys „General History of Music" aus.

Seit 1779 arbeitete Forkel dann an seiner eigenen Darstellung, einer „Allgemeinen Geschichte der Musik“, deren erster Band 1788 herauskam. Schon zehn Jahre zuvor, nämlich 1778, hatte Forkel im zweiten Band seiner „Musikalisch-kritischen Bibliothek" Hawkins „General History“, im dritten Band von 1779 Burneys Pendant einer sehr ausführlichen Rezension unterzogen: Für das weitgehend uneingeschränkte Lob von Hawkins „General History“ braucht Forkel 63 Seiten, für einen vorgeblichen Verriss von Burneys erstem Band seiner Darstellung sogar 74 Seiten. ${ }^{39}$

Dieser Verriss allerdings hat seinen Gegenstand in Burneys „Tagebuch seiner musikalischen Reise", nach dem Forkel attestiert:

Schon aus dem erwähnten Tagebuch einer musikalischen Reise, welches gleichsam als ein Vorläufer der allgemeinen Geschichte der Musik von unserm Verfasser, an-

37 Forkel, 1792 (wie Anm. 32), S. V. Forkel schreibt hier, er habe vor 15 oder 16 Jahren begonnen, sich für die historische Kenntniß der Musik zu interessieren, woraus nach dem Erscheinungsjahr der Allgemeinen Literatur zurückzurechnen ist.

38 NSuUB Göttingen, Bibl.-Arch. Ausleihregister.

39 Johann Nikolaus Forkel, Musikalisch-kritische Bibliothek, Bd. 2, Gotha 1788, S. 166-229 (Hawkins) und Bd. 3, Gotha 1779, S. 117-191 (Burney). 
gesehen werden mußte, konnte man leicht bemerken, daß die wirkliche Erscheinung des Werks mehr zu fürchten, als zu wünschen war. Die ganze Art, in der ein für unsere Kunst so wichtiges Unternehmen behandelt wurde, und die Menge von nicht nur übereilten, sondern gänzlich falschen dort befindlichen Urtheilen, verrieth nicht nur schon zum voraus, daß Hr. Burney für sein Unternehmen zu arm an wahren gründlichen musikalischen Kenntnissen sey, sondern gab auch binlänglichen Grund zu befürchten, daß er einen großen Nebel von gewissen $\mathrm{Na}$ tionalvorurtheilen noch nicht genug vertrieben haben möchte, um im Stande zu seyn, wenigstens aus demjenigen Theil seiner Geschichte, welcher die neuere Zeit, und insbesondere uns Deutsche angeht, etwas besseres als eine ärgerliche Chronik zu machen. ${ }^{40}$

Diese Aussage ist in doppelter Hinsicht mutig. Denn zum Einen ist diese neuere Zeit noch gar nicht Gegenstand des ersten Bandes von Burneys "General History“, zum Anderen zeigt ein Blick in Forkels „Allgemeine Geschichte der Musik“, dass er Burneys „General History“ nicht nur als Anregung, sondern sogar als Vorlage verstanden hat. Forkel beginnt seine „Allgemeine Geschichte“ wie Burney seine „General History" bei den ältesten Zeiten: Nämlich den alten Egyptiern und führt sie bis zum Beginn des lateinischen Mittelalters: Augustinus, Boëthius, Cassiodor. Und in vielen Details der Darstellung reicht die Anlehnung so weit, dass Oliver Wiener diesen ersten Teil von Forkels „Allgemeiner Geschichte“ als ein weiträumiges Plagiat von Burneys erstem Band beschreiben kann, wobei Wiener auf rund 50 Seiten Forkels Abhängigkeit von Burney bis in einzelne Formulierungen hinein aufzeigt. ${ }^{41}$

Es wäre ein Leichtes, Forkels akademische Karriere damit zu Fall zu bringen. Doch würde man Forkels Rolle, die auch die eines Vermittlers ist, damit nicht vollständig gerecht. Wie Burney und Hawkins geht auch Forkel in seiner „Allgemeinen Geschichte der Musik“ über eine reine Chronik von Ereignissen hinaus und deutet die Musikgeschichte mit einem bestimmten Narrativ aus. Und dieser Narrativ ist einer, der, bei Burney allerdings sehr viel stärker als bei Hawkins, vom Gedanken einer historischen Evolution der Geschichte ausgeht und letztlich auf eine musikalische Heroengeschichte abzielt. Während bei Hawkins und Burney die Musikgeschichte aber ihre eigentliche Vollendung im Schaffen Händels findet, ist Forkels Held Johann Sebastian Bach, dessen Biographie Forkel 1802 im Druck erschienen ließ.42

Der Narrativ einer historischen Evolution wird in der „Allgemeinen Geschichte der Musik" unweigerlich deutlich beim Blick auf die methodische Grundlegung der

40 Forkel, 1779 (wie Anm. 39), Bd. 3, S. $118 \mathrm{f}$.

41 Oliver Wiener, Apolls Musikalische Reisen. Zum Verhältnis von System, Text und Narration in Johann Nikolaus Forkels Allgemeiner Geschichte der Musik (1788-1801), Mainz 2009, S. 229-241.

42 Johann Nikolaus Forkel, Ueber Johann Sebastian Bachs Leben, Kunst und Kunstwerke, Leipzig 1802. 
Musikgeschichte, deren anthropologische Grundlagen Forkel als Prozess zunehmender Komplexität zeichnet: Vom ersten Zustand des Menschen an, in dem dieser noch keine Unterschiede zwischen Tönen bemerken kann, wird zunächst der Rhythmus als Mittel erkannt, Töne unterhaltend zu machen; ein Zustand, der Forkel zufolge noch bei vielen Völkern seiner Gegenwart andauere. ${ }^{43}$ Die in Forkels Gegenwart zu erkennende Ausdifferenzierung der Musik lässt sich dann mit Methoden beschreiben, die sicherlich nicht zufällig jenen entsprechen, die Forkel in seinen Vorlesungen zur Theorie der Musik bereits dargelegt hat: der musikalischen Grammatik und der musikalischen Rhetorik. ${ }^{44}$

Im zweiten Band von Forkels „Allgemeiner Geschichte der Musik“, der übrigens zu keiner Zeit unter dem Verdacht eines Plagiats steht, lässt sich dann sehr deutlich die Tendenz zur Heroengeschichtsschreibung erkennen. Nahezu jeder einzelne Paragraph der Darstellung nennt eine bedeutende Person bereits in der Überschrift: Vom Mailänder Kirchenvater Ambrosius und seinen Gesängen spannt sich so ein Bogen, der über Papst Gregor bis hin zu Josquin, Jean Mouton und Heinrich Isaac führt. Ein dritter Band der „Allgemeinen Geschichte der Musik“, der bis in Forkels Gegenwart führen sollte, ist zwar angekündigt, jedoch allen Indizien zufolge niemals auch nur ernsthaft begonnen worden. ${ }^{45}$ Dennoch wäre es müßig, schon anhand der ersten beiden Bände im Detail nachweisen zu wollen, welche der Forkelschen Darstellungen bis in die gegenwärtige Musikwissenschaft hinein überlebt haben; Forkel hat musikhistorisches Wissen kanonisiert und den Kunstwert von Musik als Maßstab für die Relevanz des lauten Nachdenkens über Musik etabliert, eine Sichtweise, von der sich auch die gegenwärtige Musikwissenschaft noch keineswegs überall entschieden distanziert.

Festzuhalten aber bleibt: Die Personalunion ist ein Raum, in dem das theoretische Nachdenken über Musik an einen Punkt der Beschäftigung führt, an dem aus dem Doctor Musicae - dem Doktor der praktisch verstandenen Musik - der Universität Oxford der Doctor scientiae musicae - der Doktor der Musikwissenschaft - wird; ein seltener, aber auch heute noch verliehener und in Deutschland offiziell anerkannter akademischer Titel. In der Person des Göttinger akademischen Musikdirektors Forkel verbinden sich dabei zwei Perspektiven: die theoretische, die ihre Wurzeln nicht zuletzt auch im Musikunterricht der deutschen Lateinschulen hat, und die historische, die von Großbritannien aus nach Göttingen gefunden hat. Für den umgekehrten Weg der theoretischen Perspektive gibt es bislang keine ganz so klar

43 Johann Nikolaus Forkel, Allgemeine Geschichte der Musik, Bd. 1, Leipzig 1788, S. 5 f.

44 Forkel, 1788 (wie Anm. 43), S. $36 f$.

45 F. L. Cr., Johann Nikolaus Forkel, in: Zeitgenossen. Ein biographisches Magazin für die Geschichte unserer Zeit 4 (1819), S. 123-136, hier S. 136. 
erkennbaren Konturen: Hier sind noch in erheblichem Maße Forschungsarbeiten zu leisten.

Der Blick auf die Musik als Wissenschaft im Raum der Personalunion bliebe freilich in jedem Fall unvollständig ohne einen Blick auf diejenige Person, die es Forkel mutmaßlicherweise überhaupt erst ermöglicht hat, die Schriften von Hawkins und Burney zu lesen. Wenn die Universität Göttingen als Universität der Personalunion auch einen starken Bezug zur englischen Sprache hatte, gibt es doch Anlass zu zweifeln, ob Forkel im Englischen sattelfest genug für die Lektüre war. Ins Spiel kommt damit Margareta Dorothea - genannt Meta - Liebeskind, geborene Wedekind, Tochter des Göttinger Philosophieprofessors Rudolf Wedekind, seit 1778 Forkels Klavierschülerin, seit 1782 seine Ehefrau, von der er sich 1794 nach sehr turbulenten Ehejahren wieder scheiden ließ. ${ }^{46}$ Meta Liebeskind war ohne Zweifel in der englischen Sprache sehr begabt. Sie übersetzte für Georg Forster zahlreiche Bände in einer Geschwindigkeit, die die in der Sekundärliteratur vertretene Aussage, sie sei der Simultanübersetzung fähig, als durchaus plausibel erscheinen lässt. Zur Turbulenz der Forkelschen Ehe gehört, dass Meta eher selten in Göttingen anwesend war; es dauerte nach der Eheschließung sogar eine erhebliche Zeit, bis das Ehepaar überhaupt eine gemeinsame Wohnung beziehen konnte. Legt man nun aber die Zeiten von Metas Anwesenheit in Göttingen und das Ausleihregister der Universitätsbibliothek nebeneinander, zeigt sich recht deutlich, dass Forkel die „General Histories“ von Hawkins und Burney nur dann ausgeliehen hat, wenn Meta in Göttingen war - vielleicht war sie also seine Übersetzerin. Und vielleicht liegt darin auch ein Grund, warum Forkels während der Ehe entstandener erster Band der „Allgemeinen Geschichte der Musik" ein so weiträumiges Plagiat von Burneys erstem Band der "General History“ sein konnte, während der zweite Band nicht nur kein Plagiat mehr ist, sondern auch einem anderen Konzept der Darstellung folgt. Ohne die Anregung des Doctor Musicae der Universität Oxford Charles Burney hätte Forkel demnach nicht zu einer historischen Beschäftigung mit Musik gefunden, die ihn in den Stand versetzte, zum Doctor honoris causa der Universität Göttingen werden zu können. Ohne die starke Frau an seiner Seite aber möglicherweise auch nicht.

46 Andreas Waczkat, Forkel's Muse. Meta Forkel-Liebeskind and how Johann Nikolaus Forkel Read Works in English, in: Göttinger Händel-Beiträge 15 (2014) S.196-205.. 